\title{
Nasal Packing in the Emergency Department: A Practical Review for Emergency Providers
}

\author{
Quincy K Tran (1D ${ }^{1,2}$ \\ Jeremy Barnett ${ }^{3}$ \\ Francis O'Connell ${ }^{3}$ \\ Brian D'Anza ${ }^{4}$ \\ Ali Pourmand ${ }^{3}$
}

'Department of Emergency Medicine, University of Maryland School of Medicine, Baltimore, MD, USA; ${ }^{2}$ Program in Trauma, The R Adams Cowley Shock Trauma Center, University of Maryland School of Medicine, Baltimore, MD, USA;

${ }^{3}$ Department of Emergency Medicine, George Washington University School of Medicine and Health Sciences, Washington, DC, USA; ${ }^{4}$ Department of Otolaryngology - Head and Neck Surgery, Case Western Reserve University School of Medicine, Cleveland, $\mathrm{OH}, 44106$, USA
Correspondence: Quincy K Tran Email qtran@som.umaryland.edu

\begin{abstract}
We performed a narrative review of epistaxis management in the emergency department. First, we examined the pathophysiology, the current types of treatment that are available to emergency clinicians. When nasal packing is indicated, we examined the efficacy of nasal packing in addition to other topical treatment such as tranexamic acid and the evidence of prophylactic antibiotics. We detailed current studies involving tranexamic acid and prophylactic antibiotics for nasal packing. Finally, we introduced an epistaxis clinical care pathway, based on current evidence, to aid emergency clinicians with their clinical decision-making processes.
\end{abstract}

Keywords: epistaxis, tranexamic acid, nasal packing, clinical care pathway

\section{Introduction}

Epistaxis has a lifetime incidence of $60 \%$ in the general population and of those who develop epistaxis, about $10 \%$ will visit the emergency department (ED) for treatment. ${ }^{1}$ Current first-line treatment has remained the same for the past 20 years, which requires sitting the patient up and tilting the head forward while pinching the nasal septum to apply pressure on the Kiesselbach's plexus for 10-15 minutes. ${ }^{1}$ Bleeding from the Kiesselbach's plexus (anterior epistaxis) accounts for around $80 \%$ of all cases. ${ }^{2}$ Causes of epistaxis include nose picking (trauma), temperature change, allergic rhinitis, specific medications such as (anticoagulation), and idiopathic sources. ${ }^{3}$

Anterior epistaxis accounts for most of the epistaxis seen in the ED, which usually has a bleeding source at the anterior inferior septum in Little's area. ${ }^{4}$ Management of active epistaxis in the ED usually starts with 10-15 minutes of direct pressure followed by attempting to identify a source of bleeding via anterior rhinoscopy. ${ }^{5}$ Inspection for the site of bleeding requires removal of any hematoma from the applied pressure, which can be done by nose blowing, suction or direct removal. ${ }^{6}$ Anterior epistaxis is usually a less severe form of epistaxis with a wide range of treatment modalities that will be discussed later in this review.

Posterior epistaxis' source of bleeding is linked to the branches of the internal maxillary artery which is the sphenopalatine and the posterior ethmoid artery originating from the ophthalmic artery. ${ }^{7,8}$ It is often more serious than anterior epistaxis and often requires transfer or referral to Otolaryngologists (ENT). ${ }^{7}$ Continued bleeding after direct pressure, especially when the bleeding spreads into the posterior pharynx increases the likelihood of posterior epistaxis. ${ }^{3}$ Posterior epistaxis is twice as likely to require nasal packing and a longer hospital stay. ${ }^{8}$ Posterior epistaxis' source of bleeding is usually undefined when using rhinoscopy, contributing to the increased need of packing to slow 
bleeding before more direct interventions can be used. ${ }^{8}$ Rigid endoscopy is often needed to locate the source of bleeding in posterior epistaxis with a success rate of $80-94 \%{ }^{9}{ }^{9}$

Nasal packing continues to be a follow-up treatment if bleeding fails to stop after 15 minutes of pressure, with $20 \%$ of ED visits requiring nasal packing. ${ }^{3}$ Nasal Packing is conducted more often if the source of bleeding is not identified by anterior rhinoscopy or if the amount of bleeding obscures the site of the bleed. ${ }^{4}$ Identification of a bleeding site leads to cauterization with silver nitrate and packing if the bleeding persists. Up to $52 \%$ of the patients who have packing will also have nasal cauterization. ${ }^{3}$ Nasal packing typically can take two different forms, absorbable and nonabsorbable, which will be detailed later in this article. Nasal packing has limitations including reduced ventilation, sleep apnea, and general patient discomfort. Nasal packing encompasses a variety of packing material and additive medications for treatment. The purpose of this article is to perform a narrative review of epistaxis treatment options in the ED, focusing on nasal packing. This includes a description of when nasal packing is necessary, options for nasal packing, and duration of packing among other topical measures such as tranexamic acid. The review will address packing recommendations in both posterior and anterior epistaxis medical cases.

\section{Methods}

PubMed and SCOPUS searches were conducted using a combination of the keywords "Epistaxis/therapy", "nasal packing", "and emergency department" using Medical Subject Headings (MeSH) in PubMed. The references of included studies were also reviewed to identify additional sources. Furthermore, additional references were added at the author's discretion. The initial literature search identified 46 articles, of which 30 articles were included in this review. We included studies such as letters, perspectives, clinical guidelines, and retrospective studies. Articles were not included if they were not primarily in English or did not have an English translation, if they focused on pediatric patients or contained preliminary/unpublished results. This article is based on previously conducted studies and does not contain any studies with human participants or animals performed by any of the authors.

\section{Results}

\section{Cauterization}

Cauterization and nasal packing continue to be subsequent treatments for epistaxis in the ED when direct digital compression fails to stop the bleeding. Cauterization and nasal packing are a key interventional step in numerous clinical care pathways (CCP) published in both the International Journal of Otolaryngology and American Journal of Otolaryngology. ${ }^{10,11}$ Richardson et al wrote a specific checklist in 2021 to provide ED clinicians with a clinical care pathway to aid in reducing the amount of inconsistencies between EM and Otolaryngology treatment, the clinical care pathway led to a $61 \%$ decrease of transfer for epistaxis and a reduction of healthcare cost. ${ }^{10}$ Cauterization as a treatment option is more viable if the source of bleeding can be identified, and provides economic advantages compared to the cost of nasal packing., ${ }^{2,10,11}$ Cauterization should be used if the location of the bleed can be identified in cases of anterior epistaxis. ${ }^{12}$ If the bleeding site is not identified or cauterization is not available, nasal packing is required, which leads to them being closely related in the clinical care pathway.

Cautery can cause several side effects such as recurrent epistaxis, failure to stop epistaxis, crusting, and discoloration of nasal mucosa. ${ }^{13}$ A more serious complication is nasal septal perforation but can be reduced by limiting cauterization to both sides of the same area of the septum. ${ }^{3}$ Blind cauterization can lead to an increased risk of ulceration and destruction of the nasal septum by silver nitrate or electrical current. ${ }^{6}$

\section{Electrical and Chemical Cautery}

Cauterization can be performed using electrical energy or topical application of chemicals. The most common chemical agent is silver nitrate, which is coated on tips of plastic sticks. ${ }^{4,13}$ As previously mentioned, identification of the site of the bleed is needed before cauterization, as well as local anesthetics, which can be direct aerosolized or application of a soaked cotton ball with the agent. ${ }^{3}$ Cauterization using silver nitrate can require at least of 30 seconds of direct contact with the source of the bleed. $^{14,15}$ Electrical cauterization can be achieved by monopolar or bipolar diathermy with bipolar being the more commonly used technique. ${ }^{16}$ Electrical cauterization has an increased efficacy with a failure rate of $14.5 \%$ compared to chemical cautery with a failure rate of $35.1 \% .{ }^{16}$ Bipolar cautery was compared to nasal packing by Henderson in a cohort study in the UK; in the study use of Bipolar cautery led to a significant drop in hospital admission from $62 \%$ to $37 \% .{ }^{17}$ Limited evidence showed that electrical cautery is not associated with higher patient discomfort or complications. ${ }^{16}$ 


\section{Nasal Packing}

Nasal packing is the treatment modality after the failure of digital compression and inability to locate the bleed with anterior rhinoscopy that is required for cautery. ${ }^{3}$ Sethi et al conducted a retrospective review of Nationwide Emergency Department Sample (NEDS) from 2009 to 2011 breaking down nasal packing in the ED, 52.2\% of nasal packing was conducted with cautery, $40.5 \%$ was anterior packing alone, and $7.3 \%$ was anterior and posterior packing. ${ }^{18}$ Nasal packing was found to be used slightly more in elderly patients as a treatment after digital compression failed to stop the bleeding ( $88.4 \%$ compared to $86.7 \%$ in the general population), with the average age of those receiving nasal packing in the ED being 64.6 years. ${ }^{18,19}$

The placement, removal and presence of nasal packing is usually uncomfortable for the patient. ${ }^{3}$ Nasal packing requires a minimum of 24 hours in place with at least 48 hours needed for higher risk patients. Studies have shown that shorter packing durations were associated with treatment failures. ${ }^{20,21}$ Nasal packing complications include displacement of nasal septum causing airway obstruction, development of a sinus infection, and posterior dislocation with possible aspiration of nasal packs. ${ }^{9,20}$ In patients with nasal septal deviations, there is a possibility for mucosal abrasions or major trauma to septal ridges. Contraindications for nasal packing are facial, nasal bone, or basilar skull fractures. ${ }^{4}$

\section{Nasal Packing Material}

Nasal packing material is divided into two major categories of absorbable and non-absorbable (removal needed), with specific packs made for anterior and posterior epistaxis made from a variety of different manufacturers. ${ }^{3,4,7}$ Non-absorbable packing was the traditional packing option using cotton stripping impregnated petroleum jelly, inflatable balloons, and polyvinyl alcohol. ${ }^{6,7}$ The major disadvantage of non-absorbable packing material is pain on removal. ${ }^{6}$ Absorbable packing material is preferred for patients with bleeding disorders. It also involves decreased pain during placement and avoids the painful process of removing the packing material. ${ }^{3}$ Absorbable packing should be attempted before non-absorbable packing due to patient comfort; absorbable packing materials include surgicel, thrombinsoaked gelfoam, and floseal. ${ }^{10}$ Absorbable packing can be used in both anterior and posterior epistaxis, though it is more typically involved in anterior epistaxis. ${ }^{2}$

\section{Posterior and Anterior Nasal Packing}

Posterior packing introduces compressive material to the nasopharynx and posterior nasal cavity using a balloon or Foley catheter. ${ }^{6,12}$ This can cause an excessive pressure and consequently cause a necrosis of the nasal septum. Since cautery treatment usually requires visualization of location of the bleeding source, posterior nasal packing is the main treatment modality for posterior epistaxis in the ED. ${ }^{3,4}$ Posterior packing is a painful procedure that will sometimes be performed by ED clinicians in emergency situations where ENT specialist is not immediately available. All patients with posterior packing should be seen by an ENT specialist when one becomes available or be transferred to an institution that has ENT specialty. ${ }^{4,10}$ Anterior nasal packing is used when digital compression fails to stop the bleeding, and when identification of the source of the bleed cannot be identified or cautery fails to stop the bleeding. ${ }^{3,10,11}$

\section{Topical/Local Treatment}

Topical treatment modalities attempt to establish hemostasis as the other current treatments do as well. Tranexamic acid (TXA) is an antifibrinolytic agent that is applied in the case of anterior epistaxis, which acts as a competitive inhibitor of plasminogen activation, ${ }^{22}$ Zahed et al conducted a randomized control trial (RCT) comparing TXA on a cotton ball applied topically compared to anterior epistaxis packing with cotton pledgets soaked with epinephrine. The RCT found that hemostasis was achieved in $70 \%$ of the cases within 10 minutes of TXA application compared to $30 \%$ for anterior epistaxis packing. The study also found that rebleeding within 7 days for TXA treatment was $3 \%$ compared to $11 \%$ in the packing group. ${ }^{23}$ Zahed et al further conducted research in using the same methods of treatment in patients taking antiplatelet drugs with similar results of $71 \%$ achieving hemostasis in 10 minutes compared to $31 \%$ of the patients receiving anterior epistaxis packing. ${ }^{24}$ Birmingham et al conducted a similar study with results pointing to greater efficacy of TXA reaching hemostasis at 10 minutes, as well as a significant decrease in otolaryngologist consultations in the TXA group. ${ }^{25}$

Oxymetazoline and phenylephrine are vasoconstrictors that can be administered as a nasal spray or soaked on a cotton pledget for achieving hemostasis. ${ }^{3}$ Whitworth et al conducted a prospective study comparing topical TXA versus topical oxymetazoline in the treatment of 
anterior epistaxis. They found that TXA achieved hemostasis in $78 \%$ of the patients compared to $35 \%$ in the oxymetazoline group, with both being used as first-line therapies to avoid the use of nasal packing. ${ }^{22}$

\section{Prophylactic Antibiotics}

Nasal packing's infectious risks include local infection, bacteremia, and toxic shock syndrome (TSS) with most packs removed after 48 hours clinically having a foul smell suggesting rapid bacterial colonization of packing. ${ }^{26}$ Most studies reporting these complications involve the use of non-absorbable nasal packing. Use of prophylactic antibiotics in anterior epistaxis is a controversial source of study and practice in the last few years. ${ }^{27,28}$ Prescription of antibiotics appears to correlate with the duration of nasal packing with $37 \%$ prescribing antibiotics with $>24$ hours and $65 \%$ doing so $>48$ hours. ${ }^{28}$ Most studies done on the efficacy of systemic antibiotics used in nasal packing are small retrospective or nonrandomized studies. ${ }^{29}$ The use of antibiotics for TSS prevention has been investigated as clinical practice guidelines and studies about the subjects are limited. ${ }^{27,30}$ Interestingly, TSS has a lower incidence with anterior nasal packing ( 1 in 6060) than anaphylaxis from systemic antibiotics ( 1 in 5000). ${ }^{30}$ Findings of recent studies failed to demonstrate nasal infections in any patients regardless if they were prescribed oral antibiotics for systemic prophylaxis. ${ }^{28,29}$ The use of antibiotics is leading to an increase of resistant organisms in the hospital setting. ${ }^{27}$ Crohn's study in 2015 pointed to the need of antibiotics only in patients who are immunosuppressed when receiving anterior nasal packing. ${ }^{29}$

\section{Discussion}

Nasal packing is an effective, safe treatment option after nasal compression fails to achieve hemostasis or when the bleeding site is not identified. Implementing a clinical care pathway and training emergency physicians in the skill sets on the clinical care pathway would lower rates of transfer to another hospital, reduce variability, and improve treatment outcomes for the patient. ${ }^{10,11}$ Consequently, effective care in the ED, while reducing cost to the patient and the hospital, as well as decreasing length of stay in the ED, improves patient outcome and satisfaction.

Several clinical care pathways suggest that tranexamic acid (TXA) and other topical treatments provide better patient comfort and faster treatment. ${ }^{3,10}$ Richardson et al from University Hospitals suggested a clinical care pathway that uses oxymetazoline-soaked cotton pledget as a treatment option before using a nasal speculum to identify bleeding location, and prior to using absorbable packing material for anterior epistaxis. ${ }^{10}$ Due to evolving literature, recently University Hospitals updated their clinical care pathway to include TXA (Figure 1). An updated search shows that clinical care pathways that were published in the last few years, ${ }^{3,10,11}$ compared to clinical guidelines from as recently as 5 years ago showed a preference to use more topical treatments than absorbable nasal packing due to topical treatments' higher clinical success and patient comfort.

Topical treatment, specifically with $\mathrm{TXA}^{22-25}$ is a promising field for future investigations because it promises to be faster and more effective for epistaxis in the ED. Tranexamic acid (TXA) is considered an essential medication by the World Health Organization (WHO) because it is relatively cheap, it is commonly available in EDs in the United States. ${ }^{24}$ Currently, the literature comparing the use of TXA versus oxymetazoline, or regular nasal packing is limited. However, two previous studies $^{23,25}$ demonstrated the efficacy of TXA when they showed that topical treatment with TXA was able to achieve epistaxis within 10 minutes in $70 \%$ of the patients and was associated with superior patient comfort. However, the dosage and form of TXA are still debated as these studies used both topical treatment and packing in the form of TXA-soaked cotton pledgets. ${ }^{23-25}$

The majority of physicians prescribe prophylactic antibiotics for epistaxis nasal packing to reduce the chance of TSS, and sino-nasal and middle ear infection. ${ }^{28,29}$ No studies exist that demonstrate a case where TSS or middle ear infection occurred when antibiotics were not prescribed. ${ }^{29}$ Antibiotics are prescribed more when nonabsorbable packing is used. ${ }^{30}$ Antibiotic resistant microbes continue to be a major area of concern and should be weighed against the probability of infection when nasal packing is administered. ${ }^{27,29}$ Current trends of antibiotic use depend on the duration of nasal packing as there are no present guidelines. ${ }^{3,28}$ Depending on future research, a new clinical pathway might center on prophylactic antibiotic use on immunocompromised patients rather than packing duration. ${ }^{29}$

The majority of epistaxis episodes that arrive in the ED are handled by emergency clinicians, with the varied methods of TXA, nasal packing, and cautery achieving hemostasis. ${ }^{3,9}$ The location of the source of bleeding is 


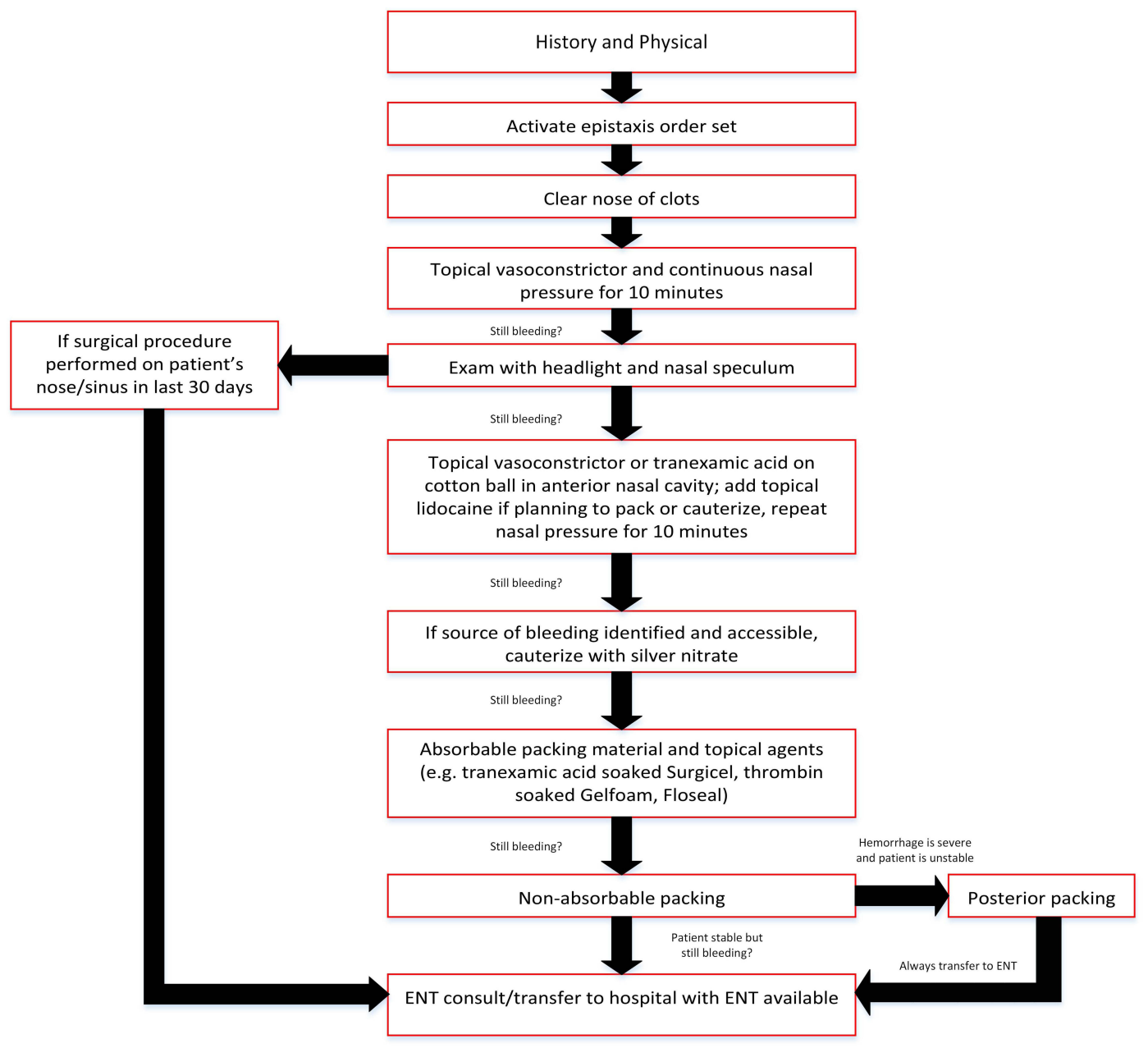

Figure I Epistaxis clinical care pathway.

Abbreviation: ENT, otolaryngology (ear nose throat).

important for determining whether packing, cautery, or topical treatment will be the most appropriate treatment. $^{3,9,12}$ A stepwise approach in the management of epistaxis is needed. Cutting down on consultations provides a decrease in health care cost and increases patient satisfaction if there is clinical success in the ED. Posterior packing will lead to an ENT consultation due to the more serious causes, lower chance of locating the bleed, and lower clinical success of conservative management. ${ }^{3,10}$ Overall, the key is using the right intervention at the right time, whether it is packing or otherwise. Clinical care pathways can assist in this determination, but the clinicians' clinical decisionmaking will always remain paramount.

\section{Limitations}

There are several limitations in our literature review. Many of the studies contain small sample sizes with results that are not statistically significant. The number of studies that compare newer treatment modalities with nasal packing are limited, leading to the inability to complete a formal systematic review according to PRISMA guidelines. There is no consensus on the duration needed for packing to provide clinical success, studies range from several hours to several days with no clear statistical advantage.

\section{Conclusion}

Epistaxis will continue to be a constant cause of visits in the ED patient population due to its numerous causes and high incidence rate in the population. Emergency clinicians have the capability to deal with most epistaxis cases in the ED. Nasal packing is a viable treatment modality in epistaxis where bleeding sites are unidentified and for posterior epistaxis prior to a consultation with ENT specialists. Absorbable packing should be the packing material of choice due to increased patient comfort and stopping the need for a follow- 
up visit for packing removal. An established clinical pathway provides ED clinicians a clear clinical decision-making guide in the care of epistaxis to improve clinical outcomes. Further research is needed on the efficacy of TXA as a main treatment modality for epistaxis in the ED. The combination of the availability of TXA and the high quantity of epistaxis cases in the ED makes this a viable place for future research. Prophylactic antibiotics are still heavily prescribed and further research is needed to know what patient population needs these medications for clinical success.

\section{Author Contributions}

All authors made a significant contribution to the work reported, whether that is in the conception, study design, execution, acquisition of data, analysis and interpretation, or in all these areas; took part in drafting, revising or critically reviewing the article; gave final approval of the version to be published; have agreed on the journal to which the article has been submitted; and agree to be accountable for all aspects of the work.

\section{Funding}

The authors did not receive any source of funding, internal or commercial, for the work of this manuscript.

\section{Disclosure}

The authors declared no conflicts of interest.

\section{References}

1. Sowerby L, Rajakumar C, Davis M, Rotenberg B. Epistaxis first-aid management: a needs assessment among healthcare providers. J Otolaryngol Head Neck Surg. 2021;50:7. doi:10.1186/s40463-02000485-8

2. Meccariello G, Georgalas C, Montevecchi F, et al. Management of idiopathic epistaxis in adults: what's new? Acta Otorhinolaryngol Ital. 2019;39:211-219. doi:10.14639/0392-100X-2155

3. Tunkel DE, Anne S, Payne SC, et al. Clinical practice guideline: nosebleed (epistaxis). Otolaryngol Head Neck Surg. 2020;162:S1S38. doi:10.1177/0194599819890327

4. Seikaly H, Solomon CG. Epistaxis. N Engl J Med. 2021;384:944-951. doi:10.1056/NEJMcp2019344

5. Wei W, Lai Y, Zang C, et al. A blind area of origins of epistaxis: technical or cognitive? Eur Arch Otorhinolaryngol. 2018;275:1501-1505. doi:10.1007/s00405-018-4983-7

6. Womack JP, Kropa J, Jimenez Stabile M. Epistaxis: outpatient management. Am Fam Physician. 2018;98:240-245.

7. Yüksel A, Kurtaran H, Kankiliç ES, Ark N, Uğur KS, Gündüz M. Epistaxis in geriatric patients. Turk J Med Sci. 2014;44:133-136. doi:10.3906/sag-1301-58

8. McClurg SW, Carrau R. Endoscopic management of posterior epistaxis: a review. Acta Otorhinolaryngol Ital. 2014;34:1-8.

9. Beck R, Sorge M, Schneider A, Dietz A. Current approaches to epistaxis treatment in primary and secondary care. Dtsch Arztebl Int. 2018;115:12-22. doi:10.3238/arztebl.2018.0012
10. Richardson C, Abrol A, Cabrera CI, et al. The power of a checklist: decrease in emergency department epistaxis transfers after clinical care pathway implementation. Am J Otolaryngol. 2021;42:102941. doi:10.1016/j.amjoto.2021.102941

11. Traboulsi H, Alam E, Hadi U. Changing trends in the management of epistaxis. Int J Otolaryngol. 2015;2015:263987. doi:10.1155/2015/ 263987

12. Bequignon E, Vérillaud B, Robard L, et al. Guidelines of the French Society of Otorhinolaryngology (SFORL). First-line treatment of epistaxis in adults. Eur Ann Otorhinolaryngol Head Neck Dis. 2017;134:185-189. doi:10.1016/j.anorl.2016.09.008

13. Limbrick J, Takwoingi YM. Bilateral nasal septal chemical cautery: a safe and effective outpatient procedure for control of recurrent epistaxis, our experience in 134 patients. Eur Arch Otorhinolaryngol. 2019;276:1845-1848. doi:10.1007/s00405-019-05389-6

14. Bastianpillai J, Saxby C, Coyle P, Armstrong A, Mohamid W, Mochloulis G. Evaluating nasal cautery techniques in epistaxis. J Laryngol Otol. 2019;133:1-5. doi:10.1017/S0022215118002293

15. Ha JF, Hodge JC, Lewis R. Comparison of nasendoscopic-assisted cautery versus packing for the treatment of epistaxis. ANZ J Surg. 2011;81:336-339. doi:10.1111/j.1445-2197.2010.05602.x

16. Mcleod RWJ, Price A, Williams RJ, Smith ME, Smith M, Owens D. Intranasal cautery for the management of adult epistaxis: systematic review. $J$ Laryngol Otol. 2017;131:1056-1064. doi:10.1017/ S0022215117002043

17. Henderson AH, Larkins A, Repanos C. The use of bipolar electrocautery in adult epistaxis management: using audit of one hundred and twenty-four cases to define a standardised protocol. Clin Otolaryngol. 2013;38:554-558. doi:10.1111/coa.12191

18. Sethi RKV, Kozin ED, Abt NB, Bergmark R, Gray ST. Treatment disparities in the management of epistaxis in United States emergency departments. Laryngoscope. 2018;128:356-362. doi:10.1002/lary.26683

19. Zhou AH, Chung SY, Sylvester MJ, et al. To pack or not to pack: inpatient management of epistaxis in the elderly. Am J Rhinol Allergy. 2018;32:539-545. doi:10.1177/1945892418801259

20. Spielmann PM, Barnes ML, White PS. Controversies in the specialist management of adult epistaxis: an evidence-based review. Clin Otolaryngol. 2012;37:382-389. doi:10.1111/coa.12024

21. Shargorodsky J, Bleier BS, Holbrook EH, et al. Outcomes analysis in epistaxis management: development of a therapeutic algorithm. Otolaryngol Head Neck Surg. 2013;149:390-398. doi:10.1177/ 0194599813492949

22. Whitworth K, Johnson J, Wisniewski S, Schrader M. Comparative effectiveness of topically administered tranexamic acid versus topical oxymetazoline spray for achieving hemostasis in epistaxis. J Emerg Med. 2020;58:211-216. doi:10.1016/j.jemermed.2019.11.038

23. Zahed R, Moharamzadeh P, Alizadeharasi S, Ghasemi A, Saeedi M. A new and rapid method for epistaxis treatment using injectable form of tranexamic acid topically: a randomized controlled trial. $\mathrm{Am}$ J Emerg Med. 2013;31:1389-1392. doi:10.1016/j.ajem.2013.06.043

24. Zahed R, Mousavi Jazayeri MH, Naderi A, Naderpour Z, Saeedi M. Topical tranexamic acid compared with anterior nasal packing for treatment of epistaxis in patients taking antiplatelet drugs: randomized controlled trial. Acad Emerg Med. 2018;25:261-266. doi:10.1111/acem. 13345

25. Birmingham AR, Mah ND, Ran R, Hansen M. Topical tranexamic acid for the treatment of acute epistaxis in the emergency department. $\mathrm{Am}$ J Emerg Med. 2018;36(7):1242-1245. doi:10.1016/j.ajem.2018.03.039

26. Gupta A, Agrawal SR, Sivarajan K, Gupta V. A microbiological study of anterior nasal packs in epistaxis. Indian $J$ Otolaryngol Head Neck Surg. 1999;51:42-46. doi:10.1007/BF02996844

27. Tran QK, Rehan MA, Haase DJ, Matta A, Pourmand A. Prophylactic antibiotics for anterior nasal packing in emergency department: a systematic review and meta-analysis of clinically-significant infections. Am J Emerg Med. 2020;38:983-989. doi:10.1016/j. ajem.2019.11.037 
28. Murano T, Brucato-Duncan D, Ramdin C, Keller S. Prophylactic systemic antibiotics for anterior epistaxis treated with nasal packing in the ED. Am J Emerg Med. 2019;37:726-729. doi:10.1016/j. ajem.2018.12.056

29. Cohn B. Are prophylactic antibiotics necessary for anterior nasal packing in epistaxis? Ann Emerg Med. 2015;65:109-111. doi:10.1016/j.annemergmed.2014.08.011
30. Hu L, Gordon SA, Swaminathan A, Wu T, Lebowitz R, Lieberman S. Utilization of prophylactic antibiotics after nasal packing for epistaxis. $J$ Emerg Med. 2021;60:144-149. doi:10.1016/j. jemermed.2020.10.011

\section{Publish your work in this journal}

The Open Access Emergency Medicine is an international, peerreviewed, open access journal publishing original research, reports, editorials, reviews and commentaries on all aspects of emergency medicine. The manuscript management system is completely online

Submit your manuscript here: https://www.dovepress.com/open-access-emergency-medicine-journal and includes a very quick and fair peer-review system, which is all easy to use. Visit http://www.dovepress.com/testimonials.php to read real quotes from published authors. 